Interfaces and Free Boundaries 13 (2011), 105-125

DOI $10.4171 / \mathrm{IFB} / 250$

\title{
On the evolution of subcritical regions for the Perona-Malik equation
}

\author{
MARINA GHISI \\ Dipartimento di Matematica "Leonida Tonelli”, Università degli Studi di Pisa, Pisa, Italy \\ E-mail: ghisi@dm.unipi.it \\ MASSIMO GoBBino \\ Dipartimento di Matematica Applicata “Ulisse Dini”, Università degli Studi di Pisa, Pisa, Italy \\ E-mail:m.gobbino@dma.unipi.it
}

[Received 13 September 2009 and in revised form 3 July 2010]

\begin{abstract}
The Perona-Malik equation is a celebrated example of forward-backward parabolic equation. The forward behavior takes place in the so-called subcritical region, in which the gradient of the solution is smaller than a fixed threshold. In this paper we show that this subcritical region evolves in a different way in the following three cases: dimension one, radial solutions in dimension greater than one, general solutions in dimension greater than one.

In the first case subcritical regions do not shrink, that is, that they expand with a nonnegative rate. In the second case they expand with a positive rate and always spread over the whole domain after a finite time, depending only on the (outer) radius of the domain. As a by-product, we obtain a nonexistence result for global-in-time classical radial solutions with large enough gradient. In the third case we show an example where subcritical regions do not expand.

Our proofs exploit comparison principles for suitable degenerate and nonsmooth free boundary problems.
\end{abstract}

2010 Mathematics Subject Classification: 35K55, 35K65, 35R35.

Keywords: Perona-Malik equation; forward-backward parabolic equation; degenerate parabolic equation; moving domains; subsolutions and supersolutions.

\section{Introduction}

In this paper we consider the Perona-Malik equation

$$
u_{t}(x, t)-\operatorname{div}\left(\frac{\nabla u(x, t)}{1+|\nabla u(x, t)|^{2}}\right)=0 \quad \forall(x, t) \in \Omega \times[0, T),
$$

where $\Omega \subseteq \mathbb{R}^{n}$ is an open set and $T>0$. This equation is the formal gradient flow of the functional

$$
P M(u):=\frac{1}{2} \int_{\Omega} \log \left(1+|\nabla u(x)|^{2}\right) \mathrm{d} x .
$$

The convex-concave behavior of the integrand makes (1.1) a forward-backward partial differential equation of parabolic type. The forward (or subcritical) region is the set of points $(x, t)$ where $|\nabla u(x, t)|<1$, the backward (or supercritical) region is the set of points where $|\nabla u(x, t)|>1$. 
This equation was introduced by P. Perona and J. Malik [17] in the context of image denoising. They considered equation 1.1 in a rectangle $\Omega \subseteq \mathbb{R}^{2}$, with an initial condition $u(x, 0)=u_{0}(x)$ representing the grey level of a (noisy) picture, and Neumann boundary conditions. For increasing values of $t$ the functions $x \mapsto u(x, t)$ are thought to be improved versions of $u_{0}(x)$, where the improvement mostly lies in noise reduction and edge enhancing.

The heuristic idea is that small disturbances, corresponding to small values of the gradient, are smoothed out because of the diffusion which takes place in the forward regions. On the other hand, sharp edges correspond to large values of the gradient and therefore they are expected to be enhanced by the backward character of the equation in supercritical regions. This phenomenology has been actually observed in numerical experiments, which also reveal an unexpected stability (see [7, 8, 14]). The method has well known shortcomings such as the staircasing effect observed in supercritical regions (see [19, 14]), but it has none of the dramatic instabilities which are typical, for example, of the backward heat equation. This discrepancy between the practical efficacy of (1.1) and its analytical ill-posedness has been called "Perona-Malik paradox" after S. Kichenassamy's paper [16].

In the last fifteen years the paradox has been investigated in numerous papers. Several authors proved well-posedness results for approximations of (1.1) obtained via space discretization [4, 9] or convolution [5], time delay [1], fractional derivatives [13], fourth order regularization [2], simplified nonlinearities [3].

The behavior of such approximations as a suitable parameter goes to 0 is a much more challenging problem. As far as we know, results in this direction have been obtained only for the semidiscrete scheme in dimension one. In this case the present authors [9] (see also [4]) proved that, for every initial condition $u_{0} \in B V$, approximate solutions converge to a limit, and under reasonable assumptions this limit is a classical solution of $(1.1)$ inside its subcritical region. All these results motivate analysts to look for a notion of weak solution for 1.1 which exists for large classes of initial data, is reasonably stable, and to which reasonable approximations converge.

Such a notion of weak solution would represent, from the analytical point of view, a solution to the paradox. In this direction, K. Zhang [20] (see also [6, 18]) showed that the class of Lipschitz solutions is not the right one. Indeed he proved that for any nonconstant smooth initial condition, even if subcritical, the Neumann boundary value problem admits infinitely many (pathological) local-in-time Lipschitz solutions.

Classical solutions (namely solutions which are at least of class $C^{1}$ ) have also been investigated. B. Kawohl and N. Kutev [15] observed that global-in-time classical solutions exist if the initial condition is subcritical, while in [16] it is remarked that local-in-time classical solutions cannot exist unless the initial condition is very regular in its supercritical region. Moreover the authors proved in [10] that in dimension one there exists a dense set of initial data for which the Cauchy problem with Neumann boundary conditions admits a local-in-time classical solution of class $C^{2,1}$ (i.e. with two continuous derivatives with respect to space variables, and one continuous derivative with respect to time). On the other hand, such solutions cannot be global if the initial condition has a nonempty supercritical region (see [15] and [12]).

Quite surprisingly, things are not so drastic in dimension greater than one. Indeed the authors proved in [11] that global-in-time radial solutions of class $C^{2,1}$ do exist for some classes of initial data with nonempty supercritical region.

For classical solutions one can define the family of open sets

$$
I^{-}(t):=\{x \in \Omega:|\nabla u(x, t)|<1\} \quad \forall t \in[0, T) .
$$


This is the family of subcritical regions. Its behavior as $t$ varies is the object of this paper. We point out that this definition is purely local, in the sense that it does not depend on the boundary conditions. We show three situations in which subcritical regions evolve in a different way.

The one-dimensional case. In dimension one subcritical regions do not shrink, namely

$$
I^{-}(s) \subseteq I^{-}(t) \quad \text { whenever } 0 \leqslant s \leqslant t<T
$$

Similar results were already proved in [15] and in [12]. In this paper we present an alternative proof (see Theorem 2.1 based on a comparison principle, which only requires $C^{1}$ regularity, and works essentially for all equations which are the gradient flow of a nonconvex functional. This proof gives us the opportunity to show, in a simpler setting, the method which is fundamental in the next case, when the result was not known before.

This result is optimal in the sense that it may happen that the subcritical region is the same for every $t \in[0, T)$. An example is provided by the local-in-time solutions constructed in [10].

The radial case. Let us consider a radial solution of (1.1) defined in a ball or an anulus. Then (1.2) holds true also in this case. If moreover $I^{-}(0) \neq \emptyset$, then the inclusion is strict whenever $s<t$, and there exists $T_{0}>0$ such that $I^{-}(t)=\Omega$ for every $t>T_{0}$. The value of $T_{0}$ depends on the outer radius of $\Omega$, but is independent of $u$. In other words, supercritical and critical regions disappear after a finite time depending only on $\Omega$.

If the solution survives and remains regular up to $T_{0}$, then it becomes subcritical and there are no more obstructions to global existence. This is what actually happens in the classes of global-in-time radial solutions constructed in [11].

In Theorem 2.3 we show that this is not always the case. If the initial condition has a supercritical region where the gradient is large enough, and this supercritical region is surrounded by subcritical regions, then there is no $C^{1}$ classical solution with $T>T_{0}$, independently of the boundary conditions. The reason is that the maximum of $|\nabla u(x, t)|$ in this supercritical region is a function of time which cannot decrease too fast. On the other hand, the supercritical region must disappear after $T_{0}$, and thus this maximum does not have enough time to decrease from its large initial value up to 1 .

The nonradial case. Roughly speaking, in the radial case each interface between the subcritical and the supercritical region is a circle which evolves with velocity proportional to the inverse of its radius. This reminded us of the mean curvature motion, and in a first moment led us to suspect that this interface could evolve in a similar way also for nonradial solutions. In particular it seemed reasonable that this interface could evolve in such a way that supercritical regions tend to shrink, at least where they are convex.

In Theorem 2.4 we show that this is not the case. We prove indeed that there exists an initial condition $u_{0}$ in two variables with the following properties. The supercritical region of $u_{0}$ is convex in a neighborhood of the origin, and any local-in-time solution starting from $u_{0}$, independently of the boundary conditions, has a supercritical region which invades a neighborhood of the origin. In particular any such solution does not satisfy $[1.2$.

Evolution of supercritical regions. If subcritical regions expand, then supercritical regions shrink. This is true, but not obvious. Indeed it could happen that both the subcritical and the supercritical 
region expand at the expense of the critical region where $|\nabla u(x, t)|=1$. However one can prove that this is not the case. It is enough to apply the techniques of this paper to the equation obtained by reversing the time. In this way the role of subcritical and supercritical regions is just exchanged.

Connection with free boundary problems. The evolution of subcritical regions is itself a free boundary problem. Unfortunately it involves a forward-backward equation. After some variable changes we reduce ourselves to more standard situations. Roughly speaking, in the new variable $v$ we end up with equations such as

$$
v_{t}=\sqrt{v} v_{x x}, \quad v_{t}=\sqrt{v}\left\{v_{x x}+A+\text { lower order terms }\right\},
$$

where $A>0$. These equations are satisfied where $v>0$, and we are interested in the evolution of the region where $v$ is positive.

The good news is that these equations are forward parabolic. The bad news is that they are degenerate and involve a nonlinear term which is not Lipschitz continuous. This complicates things when using comparison principles.

For the first equation we show (see Theorem 2.5 that the region where $v$ is positive does not shrink in time. This is enough to treat the Perona-Malik equation in dimension one. For the second equation we show (see Theorem 2.6) that the region where $v$ is positive expands with a positive rate depending on $A$. This implies our conclusions for the radial Perona-Malik equation.

Comments. We conclude by speculating on some consequences of our results.

From the analytic point of view they show that forward-backward diffusion reveals a rich and somewhat unexpected phenomenology in dimension $n \geqslant 2$. In the one-dimensional case the situation is simple and clear: global classical transcritical (i.e., with nonempty subcritical and supercritical regions) solutions do not exist, subcritical regions do not shrink, supercritical regions do not expand but never disappear as soon as the solution is classical. In higher dimensions the opposite is true: there are examples of global classical transcritical solutions, there is no prescribed monotonicity in the evolution of subcritical and supercritical regions (except in the radial case), and supercritical regions may disappear during the evolution.

We also emphasize that the monotonicity results proved in the one-dimensional and in the radial case depend on the interaction between forward and backward regions across the critical region. In other words, it is essential that subcritical regions are defined with respect to the critical threshold $\sigma=1$ where equation 1.1 switches from forward to backward regime. There are no corresponding results for nondegenerate forward parabolic equations. It is also essential that the solution is of class $C^{1}$ in order that it actually crosses the critical threshold instead of jumping from below to above.

From the numerical point of view, things are more complex. On the one hand, it is clear that smooth solutions cannot represent an answer to the paradox. Even if they exist for a dense set of initial data (at least in the one-dimensional case), one could always argue that this dense set is not enough "generic" to be suitable for applications, or that these solutions are not stable enough with respect to small perturbations of initial data.

On the other hand, we explained in [10] that no classical solution can be neglected by a stable theory. Any such solution, indeed, is the limit of solutions of regularized problems with vanishing perturbations (in [10] this was shown formally in the case of fourth order regularization, but the procedure is quite general).

Some of the features observed in higher dimensions might look rather strange for people interested in applications. For example, it is hard to disagree with the authors of [15] when they 
state that in image enhancing "no new edges should be generated as the image evolves, nor do we want edges to disappear". If we identify edges with supercritical regions, in dimension one the Perona-Malik equation does the job. But now we know that in higher dimensions things are quite different: supercritical regions may both disappear (which means that some edges may be lost), and invade subcritical regions (which changes the shape of objects). In other words, there are examples in dimension $n \geqslant 2$ where the Perona-Malik filter does not seem to do the job. Probably these examples are not generic enough to compromise the practical efficiency of the method, but for sure they represent a serious issue when looking for a mathematically rigorous stable theory.

This paper is organized as follows. In Section 2 we state our results for the Perona-Malik equation and the related free boundary problems. In Section 3 we prove these results.

\section{Statements}

Throughout this paper we assume that $\varphi \in C^{\infty}(\mathbb{R})$ is an even function, hence in particular $\varphi^{\prime}(0)=0$. We also assume that

$$
\begin{gathered}
\varphi^{\prime \prime}(\sigma)>0 \quad \forall \sigma \in[0,1), \\
\varphi^{\prime \prime}(1)=0, \\
\varphi^{\prime \prime}(\sigma)<0 \quad \forall \sigma>1 .
\end{gathered}
$$

These assumptions imply that $\varphi^{\prime}(1)>0$ and $\varphi^{\prime \prime \prime}(1) \leqslant 0$. In some statements we also need the stronger assumption

$$
\varphi^{\prime \prime \prime}(1)<0 .
$$

These assumptions are consistent with the concrete case $\varphi(\sigma)=2^{-1} \log \left(1+\sigma^{2}\right)$ of the PeronaMalik equation. We refer to Figure 2 for the typical behavior of $\varphi^{\prime}(\sigma)$.

We consider the equation

$$
u_{t}=\operatorname{div}\left(\varphi^{\prime}(|\nabla u|) \frac{\nabla u}{|\nabla u|}\right),
$$

which is a natural generalization of 1.1 . We believe and hope that this generality simplifies the presentation, and shows more clearly which properties of the nonlinearity are essential in each step. For the sake of generality one could also weaken the regularity assumptions on $\varphi$ (we never consider more than three derivatives), replace the threshold $\sigma=1$ in (2.2) with any $\sigma_{1}$, and weaken (2.1) and 2.3 by asking only that $\varphi^{\prime \prime}$ is positive in a left-hand neighborhood of $\sigma_{1}$ and negative in a right-hand neighborhood of $\sigma_{1}$.

\subsection{Main results}

Let us state our results on the evolution of subcritical regions. The first result concerns the onedimensional case, where 2.5. reduces to

$$
u_{t}=\left(\varphi^{\prime}\left(u_{x}\right)\right)_{x}
$$

This form of the equation is suitable for $C^{1}$ solutions, because it involves only first order derivatives. When the solution is of class $C^{2,1}$, equation 2.6. can of course be rewritten as $u_{t}=\varphi^{\prime \prime}\left(u_{x}\right) u_{x x}$. 
THEOREM 2.1 Let $\varphi \in C^{\infty}(\mathbb{R})$ be a function satisfying (2.1) through (2.3). Let $x_{1} \leqslant x_{3}<x_{4}$ $\leqslant x_{2}$ and $T>0$ be real numbers. Let $u \in C^{1}\left(\left(x_{1}, x_{2}\right) \times[0, T)\right)$ be a function satisfying 2.6 in $\left(x_{1}, x_{2}\right) \times[0, T)$, and

$$
\left|u_{x}(x, 0)\right|<1 \quad \forall x \in\left(x_{3}, x_{4}\right) .
$$

Then $\left|u_{x}(x, t)\right|<1$ for every $(x, t) \in\left(x_{3}, x_{4}\right) \times[0, T)$.

The conclusion of Theorem 2.1] is the same as in [15, Theorem 4.2] and [12, Theorem 3.3]. The new proof presented in this paper allows us to eliminate both the technical assumptions on the initial condition required in [15], and assumption [2.4] which was essential in [12]. This new approach is also independent of boundary conditions.

Our second result concerns the radial case. Let $r:=|x|$ be the radial variable, and let $u(r, t)$ be a radial solution. In this case (1.1) becomes

$$
u_{t}=\left(\varphi^{\prime}\left(u_{r}\right)\right)_{r}+(n-1) \frac{\varphi^{\prime}\left(u_{r}\right)}{r},
$$

where $n$ is the space dimension. From now on we assume for simplicity that $n=2$. The general case is completely analogous.

THEOREM 2.2 Let $\varphi \in C^{\infty}(\mathbb{R})$ be a function satisfying 2.1] through 2.4 . Let $0<r_{1} \leqslant r_{3}<$ $r_{4} \leqslant r_{2}$ and $T>0$ be real numbers. Let $u \in C^{1}\left(\left(r_{1}, r_{2}\right) \times[0, T)\right)$ be a function satisfying 2.8 (with $n=2)$ in $\left(r_{1}, r_{2}\right) \times[0, T)$, and

$$
\left|u_{r}(r, 0)\right|<1 \quad \forall r \in\left(r_{3}, r_{4}\right) .
$$

Let $k_{0}:=r_{2}^{-1} \sqrt{2 \varphi^{\prime}(1)\left|\varphi^{\prime \prime \prime}(1)\right|}$, and set

$$
\mathcal{D}:=\left\{(r, t) \in\left(r_{1}, r_{2}\right) \times[0, T): r_{3}-k_{0} t<r<r_{4}+k_{0} t\right\} .
$$

Then $\left|u_{r}(r, t)\right|<1$ for every $(r, t) \in \mathcal{D}$.

In other words, this result says that in the radial case the subcritical region expands with a rate which is bounded from below by a positive constant $k_{0}$. Figure 3 shows the shape of the set $\mathcal{D}$. The slope of the slanted lines depends on $k_{0}$. It is clear that when $t>\left(r_{2}-r_{1}\right) / k_{0}$ every nonempty initial subcritical region $\left(r_{3}, r_{4}\right)$ has invaded the whole interval $\left(r_{1}, r_{2}\right)$.

The third result concerns the nonexistence of global-in-time $C^{1}$ radial solutions if the gradient of the initial condition is too large. We point out that this result is independent of the boundary conditions.

THEOREM 2.3 Let $\varphi \in C^{\infty}(\mathbb{R})$ be a function satisfying 2.1. through 2.4. Let $0 \leqslant r_{1}<r_{3}<$ $r_{4}<r_{5}<r_{2}$ and $T>0$ be real numbers, and let $u \in C^{1}\left(\left(r_{1}, r_{2}\right) \times[0, T)\right)$ be a solution of 2.8 (with $n=2$ ) such that

$$
\left|u_{r}\left(r_{3}, 0\right)\right|<1, \quad\left|u_{r}\left(r_{5}, 0\right)\right|<1, \quad u_{r}\left(r_{4}, 0\right)>1+\frac{r_{2}\left(r_{2}-r_{1}\right)}{r_{1}^{2}} \sqrt{\frac{\varphi^{\prime}(1)}{2\left|\varphi^{\prime \prime \prime}(1)\right|}} .
$$

Then

$$
T \leqslant \frac{r_{2}\left(r_{2}-r_{1}\right)}{\sqrt{2 \varphi^{\prime}(1)\left|\varphi^{\prime \prime \prime}(1)\right|}}
$$


The above result is an extension to the radial case of the nonexistence result in dimension one (first proved in [15, Theorem 3.2] with some technical assumptions on $u_{0}$, afterwards removed in [12, Theorem 5.1]). A first difference is that in dimension one the gradient is just required to exceed somewhere the threshold $\sigma=1$, while here the gradient is required to be large enough somewhere (if not, global solutions may exist, as shown in [11]). The second difference concerns quantitative aspects, namely estimates on the life span of the solution. In dimension one it was proved in [12] that $T \leqslant 2 P M\left(u_{0}\right)$, which is a bound of the life span in terms of the initial energy. Here the bound (2.11) depends only on the geometry of the domain and on the nonlinearity.

Our last result in this paper is a counterexample to the expansion of subcritical regions for nonradial solutions. Note that the condition we impose on the initial condition $u_{0}$ depends only on the Taylor expansion of $u_{0}$ of order 3 in a neighborhood of the origin.

THEOREM 2.4 Let $\varphi \in C^{\infty}(\mathbb{R})$ be a function such that $\varphi^{\prime}(1)>0$ and $\varphi^{\prime \prime}(1)=0$. Let $\Omega \subseteq \mathbb{R}^{2}$ be any open set such that $(0,0) \in \Omega$. Let $u_{0}: \Omega \rightarrow \mathbb{R}$ be any function of class $C^{3}$ such that

$$
u_{0}(x, y)=\frac{\sqrt{2}}{2} x+\frac{\sqrt{2}}{2} y+k_{1} x^{2}+k_{2} y^{2}+h_{1} x^{3}+h_{2} y^{3}+o\left(\left(x^{2}+y^{2}\right)^{3 / 2}\right)
$$

as $(x, y) \rightarrow(0,0)$. This clearly implies that $\left|\nabla u_{0}(0,0)\right|=1$. Then one can choose the parameters $k_{1}, k_{2}, h_{1}, h_{2}$ in such a way that the following two conclusions simultaneously hold true.

(1) There exist $\delta>0, a>0$, and a convex function $g:(-\delta, \delta) \rightarrow(-a, a)$, with $g(0)=0$, such that for each $(x, y)$ in the rectangle $(-\delta, \delta) \times(-a, a)$ we have the implications

$$
\begin{aligned}
& \left|\nabla u_{0}(x, y)\right|<1 \Leftrightarrow y<g(x), \\
& \left|\nabla u_{0}(x, y)\right|=1 \Leftrightarrow y=g(x), \\
& \left|\nabla u_{0}(x, y)\right|>1 \Leftrightarrow y>g(x) .
\end{aligned}
$$

(2) Let $T>0$, and let $u \in C^{2}(\Omega \times[0, T))$ be a function satisfying 2.5 and the initial condition $u(x, y, 0)=u_{0}(x, y)$ for every $(x, y) \in \Omega$. Then there exists $t_{0} \in(0, T)$ such that

$$
|\nabla u(0,0, t)|>1 \quad \forall t \in\left(0, t_{0}\right) .
$$

Figure 1 clarifies the conclusions of Theorem 2.4 above. Statement (1) says that at time $t=0$ the origin lies in the interface $y=g(x)$ which separates the subcritical and the (locally convex)
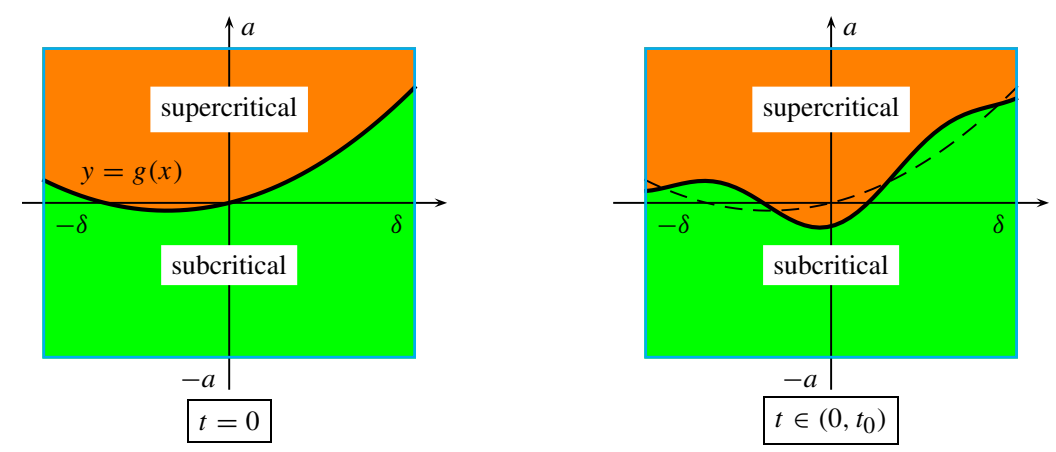

FIG. 1. At time $t=0$ the origin lies in the critical region $y=g(x)$, for subsequent times $t \in\left(0, t_{0}\right)$ the supercritical region has invaded a neighborhood of the origin. 
supercritical region. Statement (2) implies that for $t \in\left(0, t_{0}\right)$ the origin (hence also a neighborhood of the origin) has been absorbed by the supercritical region.

\subsection{Heuristics}

In this section we present simple "proofs" of Theorems 2.1 and 2.2 .

Let us start with Theorem 2.1. Let us assume that $u$ is smooth enough, and that one component of the interface between the subcritical and supercritical regions is represented by a smooth curve $(\alpha(t), t)$. Just to fix ideas, let us assume that the subcritical region lies on the left of the interface, where $x<\alpha(t)$. Taking the time derivative of the relation $u_{x}(\alpha(t), t)=1$ we obtain

$$
0=u_{x x}(\alpha(t), t) \alpha^{\prime}(t)+u_{x t}(\alpha(t), t)=u_{x x} \alpha^{\prime}(t)+\varphi^{\prime \prime}\left(u_{x}\right) u_{x x x}+\varphi^{\prime \prime \prime}\left(u_{x}\right) u_{x x}^{2},
$$

where all the partial derivatives of $u$ in the last line are computed at the point $(\alpha(t), t)$. Recalling that $\varphi^{\prime \prime}\left(u_{x}(\alpha(t), t)\right)=0$, we therefore have

$$
\alpha^{\prime}(t)=-\varphi^{\prime \prime \prime}(1) u_{x x}(\alpha(t), t) .
$$

Now $\varphi^{\prime \prime \prime}(1) \leqslant 0$, and $u_{x x}(\alpha(t), t) \geqslant 0$ because the subcritical region lies on the left of $r=\alpha(t)$. We have thus proved that $\alpha^{\prime}(t) \geqslant 0$, hence the subcritical region tends to expand.

This "proof" is not rigorous for several reasons: we assumed that $u$ is of class $C^{3}$, we assumed that the interface is a smooth curve, we divided by $u_{x x}$ which could be 0 . Nevertheless we believe that this simple argument is quite explicative.

Let us now consider Theorem 2.2 As before, we assume that the interface is given by a smooth curve $(\alpha(t), t)$, the subcritical region being on the left. Taking the time derivative of the relation $u_{r}(\alpha(t), t)=1$ we obtain

$$
\begin{aligned}
0 & =u_{r r}(\alpha(t), t) \alpha^{\prime}(t)+u_{r t}(\alpha(t), t) \\
& =u_{r r} \alpha^{\prime}(t)+\varphi^{\prime \prime}\left(u_{r}\right) u_{r r r}+\varphi^{\prime \prime \prime}\left(u_{r}\right) u_{r r}^{2}+\frac{\varphi^{\prime \prime}\left(u_{r}\right)}{\alpha(t)} u_{r r}-\frac{\varphi^{\prime}\left(u_{r}\right)}{\alpha^{2}(t)},
\end{aligned}
$$

where all the partial derivatives of $u$ in the last line are computed at the point $(\alpha(t), t)$. Recalling that $\varphi^{\prime \prime}\left(u_{r}(\alpha(t), t)\right)=0$, we have therefore

$$
\alpha^{\prime}(t)=\frac{\varphi^{\prime}(1)}{\alpha^{2}(t)} \cdot \frac{1}{u_{r r}}-\varphi^{\prime \prime \prime}(1) u_{r r}=\frac{\varphi^{\prime}(1)}{\alpha^{2}(t)} \cdot \frac{1}{u_{r r}}+\left|\varphi^{\prime \prime \prime}(1)\right| u_{r r} .
$$

Applying the inequality between the arithmetic and geometric means, we thus obtain

$$
\alpha^{\prime}(t) \geqslant \frac{2 \sqrt{\varphi^{\prime}(1)\left|\varphi^{\prime \prime \prime}(1)\right|}}{\alpha(t)} \geqslant \frac{2 \sqrt{\varphi^{\prime}(1)\left|\varphi^{\prime \prime \prime}(1)\right|}}{r_{2}} .
$$

This "proves" that the subcritical region expands with a rate which is bounded from below by a positive constant. The value of this constant is quite similar to the constant $k_{0}$ of Theorem 2.2 .

\subsection{Free boundary problems}

Our proofs of Theorems 2.1 and 2.2 rely on the following two results for free boundary problems involving degenerate and nonlipschitz parabolic equations. We state them independently because they could be interesting in themselves. 
THEOREM 2.5 Let $x_{1} \leqslant x_{3}<x_{4} \leqslant x_{2}$, and let $c_{0}>0$ and $T>0$ be real numbers. Let $g$ : $\left(0, c_{0}\right) \rightarrow(0,+\infty)$ be a continuous function. Let $v:\left(x_{1}, x_{2}\right) \times[0, T) \rightarrow \mathbb{R}$ be a function such that

(v1) $v$ is continuous in $\left(x_{1}, x_{2}\right) \times[0, T)$;

(v2) $v(x, t) \geqslant 0$ for every $(x, t) \in\left(x_{1}, x_{2}\right) \times[0, T)$;

(v3) $v(x, 0)>0$ for every $x \in\left(x_{3}, x_{4}\right)$;

(v4) the partial derivative $v_{x}(x, t)$ exists for every $(x, t) \in\left(x_{1}, x_{2}\right) \times(0, T)$;

(v5) setting

$$
\mathcal{P}:=\left\{(x, t) \in\left(x_{1}, x_{2}\right) \times(0, T): 0<v(x, t)<c_{0}\right\},
$$

we have $v \in C^{2,1}(\mathcal{P})$, and

$$
v_{t}(x, t)=g(v(x, t)) v_{x x}(x, t) \quad \forall(x, t) \in \mathcal{P} .
$$

Then $v(x, t)>0$ for every $(x, t) \in\left(x_{3}, x_{4}\right) \times[0, T)$.

THEOREM 2.6 Let $r_{1} \leqslant r_{3}<r_{4} \leqslant r_{2}$, and let $c_{0}, c_{1}, T, G, A$ be positive real numbers. Let $g:\left(0, c_{0}\right) \rightarrow(0,+\infty)$ be a continuous function such that

$$
\lim _{\sigma \rightarrow 0^{+}} \frac{g(\sigma)}{\sqrt{\sigma}}=G
$$

Let $f:\left(r_{1}, r_{2}\right) \times(0, T) \times\left[-c_{1}, c_{1}\right]^{2} \rightarrow \mathbb{R}$ be a function such that $f(r, t, 0,0)=0$ uniformly in $(r, t)$, namely

$$
\lim _{\sigma \rightarrow 0^{+}} \sup \left\{|f(r, t, p, q)|:(r, t, p, q) \in\left(r_{1}, r_{2}\right) \times(0, T) \times[-\sigma, \sigma]^{2}\right\}=0 .
$$

Let $v:\left(r_{1}, r_{2}\right) \times[0, T) \rightarrow \mathbb{R}$ be a function such that

(v1) $v$ is continuous in $\left(r_{1}, r_{2}\right) \times[0, T)$;

(v2) $v(r, t) \geqslant 0$ for every $(r, t) \in\left(r_{1}, r_{2}\right) \times[0, T)$;

(v3) $v(r, 0)>0$ for every $r \in\left(r_{3}, r_{4}\right)$;

(v4) the partial derivative $v_{r}(r, t)$ exists for every $(r, t) \in\left(r_{1}, r_{2}\right) \times(0, T)$;

(v5) setting

$$
\mathcal{P}:=\left\{(r, t) \in\left(r_{1}, r_{2}\right) \times(0, T): 0<v(r, t)<c_{0}\right\},
$$

we have $v \in C^{2,1}(\mathcal{P})$, and

$$
v_{t} \geqslant g(v)\left\{v_{r r}+f\left(r, t, v, v_{r}\right)+A\right\} \quad \forall(r, t) \in \mathcal{P} .
$$

Finally, let $\mathcal{D}$ be the set defined as in 2.9) with $k_{0}:=G \sqrt{A}$. Then $v(r, t)>0$ for every $(r, t) \in \mathcal{D}$.

\section{Proofs}

\subsection{Proof of Theorem 2.1}

We limit ourselves to proving that $u_{x}(x, t)<1$ for every $(x, t) \in\left(x_{3}, x_{4}\right) \times[0, T)$. The proof of the symmetric inequality $u_{x}(x, t)>-1$ is completely analogous.

Let us introduce some notation. Let us consider any function $h \in C^{1}(\mathbb{R})$ which is nondecreasing and such that $h(\sigma)=\varphi^{\prime}(\sigma)$ for every $\sigma \in[0,1], h(\sigma)=\varphi^{\prime}(1)$ for every $\sigma \geqslant 1$, and $h(\sigma)$ is constant 


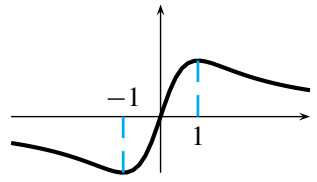

$\varphi^{\prime}(\sigma)$

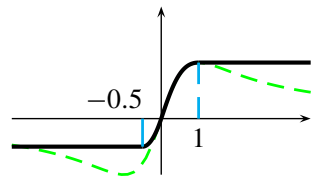

$h(\sigma)$

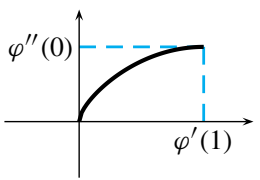

$g(\sigma)$

FIG. 2. Typical graphs of $\varphi^{\prime}, h$, and $g$.

for $\sigma \leqslant-1 / 2$. Figure 2 shows the typical graph of such a function $h$. Note that condition 2.2 is essential for the $C^{1}$ regularity of $h$.

The function $h$, as well as the function $\varphi^{\prime}$, is invertible as a function from $(0,1)$ to $\left(0, \varphi^{\prime}(1)\right)$. We can therefore define $g:\left(0, \varphi^{\prime}(1)\right) \rightarrow \mathbb{R}$ by setting

$$
g(\sigma):=\varphi^{\prime \prime}\left(h^{-1}\left(\varphi^{\prime}(1)-\sigma\right)\right) \quad \forall \sigma \in\left(0, \varphi^{\prime}(1)\right) .
$$

It is not difficult to see that the function $g$ is well defined, positive, and continuous (but not Lipschitz continuous). In the case of the Perona-Malik equation with some computations one finds that $g(\sigma)=\left(\sigma-\sigma^{2}\right)^{1 / 2}+2\left(\sigma-\sigma^{2}\right)$. Its graph is shown in Figure 2.

Let us finally set

$$
v(x, t):=\varphi^{\prime}(1)-h\left(u_{x}(x, t)\right) \quad \forall(x, t) \in\left(x_{1}, x_{2}\right) \times[0, T) .
$$

We claim that $g$ and $v$ satisfy the assumptions of Theorem 2.5 If we prove this claim, then we can conclude that $v(x, t)>0$ in $\left(x_{3}, x_{4}\right) \times[0, T)$. This is equivalent to $h\left(u_{x}(x, t)\right)<\varphi^{\prime}(1)$, which in turn is equivalent $u_{x}(x, t)<1$ in the same region.

So we only need to show that $v$ fulfils assumptions (v1) through (v5) of Theorem 2.5 .

Properties (v1) through (v3). The continuity of $v$ easily follows from the continuity of $h$ and $u_{x}$. Moreover $v(x, t) \geqslant 0$ in $\left(x_{1}, x_{2}\right) \times[0, T)$ because $h(\sigma) \leqslant \varphi^{\prime}(1)$ for every $\sigma \in \mathbb{R}$. Due to 2.7) and the fact that $\varphi^{\prime}(\sigma)<\varphi^{\prime}(1)$ when $\sigma<1$, we see that $v$ satisfies (v3).

Property (v4). It is well known that $u$ is of class $C^{\infty}$ where $\left|u_{x}(x, t)\right| \neq 1$ (because of the standard interior regularity theory for parabolic equations). Therefore the existence of the partial derivative $v_{x}(x, t)$ is trivial when $\left|u_{x}(x, t)\right| \neq 1$. The existence of $v_{x}(x, t)$ is also trivial when $u_{x}(x, t)=-1$ because $h(\sigma)$ is constant for $\sigma \leqslant 1 / 2$.

Let us now consider a point $\left(x_{0}, t_{0}\right)$ with $u_{x}\left(x_{0}, t_{0}\right)=1$. We claim that at this point $v_{x}\left(x_{0}, t_{0}\right)$ exists and is equal to 0 . Let us assume that this is not the case. Then there exists a sequence $\delta_{k} \rightarrow 0$ such that

$$
\left|\frac{\left.v\left(x_{0}+\delta_{k}, t_{0}\right)-v\left(x_{0}, t_{0}\right)\right)}{\delta_{k}}\right| \geqslant v>0 \quad \forall k \in \mathbb{N} .
$$

Up to subsequences, we can always assume that either $u_{x}\left(x_{0}+\delta_{k}, t_{0}\right)>1$ for every $k \in \mathbb{N}$, or $u_{x}\left(x_{0}+\delta_{k}, t_{0}\right) \leqslant 1$ for every $k \in \mathbb{N}$. In the first case the fraction in (3.1) is always 0 , which is incompatible with the condition stated therein. In the second case the fraction in (3.1) can be rewritten as

$$
-\frac{h\left(u_{x}\left(x_{0}+\delta_{k}, t_{0}\right)\right)-h\left(u_{x}\left(x_{0}, t_{0}\right)\right)}{\delta_{k}}=-\frac{\varphi^{\prime}\left(u_{x}\left(x_{0}+\delta_{k}, t_{0}\right)\right)-\varphi^{\prime}\left(u_{x}\left(x_{0}, t_{0}\right)\right)}{\delta_{k}} .
$$


When $\delta_{k} \rightarrow 0$, this quotient tends to $\left(\varphi^{\prime}\left(u_{x}\right)\right)_{x}\left(x_{0}, t_{0}\right)$, and we know that this derivative exists because $u$ is a solution of 2.6 of class $C^{1}$. In order to find a contradiction, it is enough to show that this derivative is equal to 0 , and this is true because it is the derivative at $x=x_{0}$ of the function $x \mapsto \varphi^{\prime}\left(u_{x}\left(x, t_{0}\right)\right)$, which attains its maximum for $x=x_{0}$.

Property (v5). Let us set

$$
\mathcal{P}:=\left\{(x, t) \in\left(x_{1}, x_{2}\right) \times(0, T): 0<v(x, t)<\varphi^{\prime}(1)\right\} .
$$

From the properties of $h$ it follows that $0<u_{x}(x, t)<1$ in $\mathcal{P}$, hence $u$ is of class $C^{\infty}$ in $\mathcal{P}$. Since $h$ and $\varphi^{\prime}$ coincide in $(0,1)$, we therefore have

$$
\begin{aligned}
v_{t} & =-h^{\prime}\left(u_{x}\right) u_{x t}=-h^{\prime}\left(u_{x}\right)\left(\varphi^{\prime}\left(u_{x}\right)\right)_{x x}=-\varphi^{\prime \prime}\left(u_{x}\right)\left(h\left(u_{x}\right)\right)_{x x}=\varphi^{\prime \prime}\left(u_{x}\right)\left(-h\left(u_{x}\right)\right)_{x x} \\
& =\varphi^{\prime \prime}\left(u_{x}\right)\left(\varphi^{\prime}(1)-h\left(u_{x}\right)\right)_{x x}=\varphi^{\prime \prime}\left(u_{x}\right) v_{x x}
\end{aligned}
$$

in $\mathcal{P}$. Moreover in $\mathcal{P}$ we can express $u_{x}$ as a function of $v$ as $u_{x}=h^{-1}\left(\varphi^{\prime}(1)-v\right)$. In conclusion

$$
v_{t}=\varphi^{\prime \prime}\left(u_{x}\right) v_{x x}=\varphi^{\prime \prime}\left(h^{-1}\left(\varphi^{\prime}(1)-v\right)\right) v_{x x}=g(v) v_{x x},
$$

which proves that $v$ satisfies (v5).

\subsection{Proof of Theorem 2.2}

The argument is similar to the proof of Theorem 2.1. We define $h, g$, and $v$ as in that proof, and we claim that the assumptions of Theorem 2.6 are satisfied.

Properties of $g$. As in the proof of Theorem 2.1 it is quite easy to show that $g:\left(0, \varphi^{\prime}(1)\right) \rightarrow$ $(0,+\infty)$ is a continuous function. It remains to compute the limit in $(2.13)$. Since we deal with positive functions, we can square the numerator and the denominator. Applying the change of variable $\tau:=h^{-1}\left(\varphi^{\prime}(1)-\sigma\right)$ and de L'Hôpital's rule, we therefore have

$$
\begin{aligned}
\lim _{\sigma \rightarrow 0^{+}} \frac{[g(\sigma)]^{2}}{\sigma} & =\lim _{\sigma \rightarrow 0^{+}} \frac{\left[\varphi^{\prime \prime}\left(h^{-1}\left(\varphi^{\prime}(1)-\sigma\right)\right)\right]^{2}}{\sigma}=\lim _{\tau \rightarrow 1^{-}} \frac{\left[\varphi^{\prime \prime}(\tau)\right]^{2}}{\varphi^{\prime}(1)-\varphi^{\prime}(\tau)} \\
& =\lim _{\tau \rightarrow 1^{-}} \frac{2 \varphi^{\prime \prime}(\tau) \varphi^{\prime \prime \prime}(\tau)}{-\varphi^{\prime \prime}(\tau)}=-2 \varphi^{\prime \prime \prime}(1)=2\left|\varphi^{\prime \prime \prime}(1)\right|,
\end{aligned}
$$

which proves 2.13 with $G:=\sqrt{2\left|\varphi^{\prime \prime \prime}(1)\right|}$.

Properties of $v$. The proof of (v1) through (v4) is analogous to the proof of the corresponding properties in Theorem 2.1. In order to prove (v5), let us consider the set $\mathcal{P}$ defined in analogy with 3.2). As in the previous case we have $0<u_{r}(r, t)<1$ in this set, hence $v$ is regular and satisfies

$$
v_{t}=-h^{\prime}\left(u_{r}\right) u_{r t}=-\varphi^{\prime \prime}\left(u_{r}\right)\left[\left(\varphi^{\prime}\left(u_{r}\right)\right)_{r}+\frac{\varphi^{\prime}\left(u_{r}\right)}{r}\right]_{r} .
$$

Once again $\varphi^{\prime \prime}\left(u_{r}\right)=\varphi^{\prime \prime}\left(h^{-1}\left(\varphi^{\prime}(1)-v\right)\right)=g(v)$. Moreover

$$
\left[\left(\varphi^{\prime}\left(u_{r}\right)\right)_{r}+\frac{\varphi^{\prime}\left(u_{r}\right)}{r}\right]_{r}=\left(\varphi^{\prime}\left(u_{r}\right)\right)_{r r}+\frac{\left(\varphi^{\prime}\left(u_{r}\right)\right)_{r}}{r}-\frac{\varphi^{\prime}\left(u_{r}\right)}{r^{2}}=-v_{r r}-\frac{v_{r}}{r}-\frac{\varphi^{\prime}(1)}{r^{2}}+\frac{v}{r^{2}} .
$$


Plugging these identities into $(3.3)$ we obtain

$$
v_{t}=g(v)\left\{v_{r r}+\frac{v_{r}}{r}-\frac{v}{r^{2}}+\frac{\varphi^{\prime}(1)}{r^{2}}\right\} \geqslant g(v)\left\{v_{r r}+f\left(r, t, v, v_{r}\right)+A\right\},
$$

where

$$
f(r, t, p, q):=\frac{q}{r}-\frac{p}{r^{2}}, \quad A:=\frac{\varphi^{\prime}(1)}{r_{2}^{2}} .
$$

We have thus proved that $v$ satisfies the differential inequality 2.15$)$ in $\mathcal{P}$ with a constant $A>0$ and a function $f(r, t, p, q)$ satisfying 2.14).

Conclusion. From Theorem 2.6 we deduce that $v$ is positive in the region $\mathcal{D}$ defined by (2.9) with $k_{0}=G \sqrt{A}=r_{2}^{-1} \sqrt{2 \varphi^{\prime}(1)\left|\varphi^{\prime \prime \prime}(1)\right|}$, which in turn implies that $u_{r}<1$ in the same region. The proof of the symmetric inequality $u_{r}>-1$ is completely analogous.

\subsection{Proof of Theorem 2.3}

Assume that there exists a solution with

$$
T>T_{0}:=\frac{r_{2}\left(r_{2}-r_{1}\right)}{\sqrt{2 \varphi^{\prime}(1)\left|\varphi^{\prime \prime \prime}(1)\right|}} .
$$

Set

$$
M(t):=\max \left\{u_{r}(r, t): r \in\left[r_{3}, r_{5}\right]\right\} \quad \forall t \in[0, T) .
$$

Due to the first two inequalities in 2.10 we know that the subcritical region is nonempty at time $t=0$. Applying Theorem 2.2 we find that the subcritical region expands, and coincides with the whole interval $\left(r_{1}, r_{2}\right)$ as soon as

$$
t>\frac{r_{2}-r_{1}}{k_{0}}=T_{0}
$$

In particular this means that

$$
M\left(T_{0}\right) \leqslant 1
$$

On the other hand we claim that

$$
M(t) \geqslant M(0)-\frac{\varphi^{\prime}(1)}{r_{1}^{2}} t \quad \forall t \in\left[0, T_{0}\right] .
$$

If we prove this claim, then setting $t=T_{0}$ and exploiting the last inequality in 2.10, we find that

$$
\begin{aligned}
M\left(T_{0}\right) & \geqslant M(0)-\frac{\varphi^{\prime}(1)}{r_{1}^{2}} T_{0} \\
& >1+\frac{r_{2}\left(r_{2}-r_{1}\right)}{r_{1}^{2}} \sqrt{\frac{\varphi^{\prime}(1)}{2\left|\varphi^{\prime \prime \prime}(1)\right|}}-\frac{\varphi^{\prime}(1)}{r_{1}^{2}} \cdot \frac{r_{2}\left(r_{2}-r_{1}\right)}{\sqrt{2 \varphi^{\prime}(1)\left|\varphi^{\prime \prime \prime}(1)\right|}}=1,
\end{aligned}
$$

which contradicts 3.4 . 
Proof of 3.5. The argument is similar to the usual comparison principles. Setting for simplicity $v(r, t):=u_{r}(r, t)$, we find that $v$ is a solution of

$$
v_{t}=\varphi^{\prime \prime}(v) v_{r r}+\varphi^{\prime \prime \prime}(v) v_{r}^{2}+\frac{\varphi^{\prime \prime}(v)}{r} v_{r}-\frac{\varphi^{\prime}(v)}{r^{2}}
$$

in the subset of $\left(r_{1}, r_{2}\right) \times[0, T)$ where $|v| \neq 1$. Let us set

$$
w(t):=M(0)-\varepsilon-\frac{\varphi^{\prime}(1)}{r_{1}^{2}} t \quad \forall t \in\left[0, T_{0}\right],
$$

where $\varepsilon>0$ is small enough so that $w\left(T_{0}\right)>1$, hence $w(t)>1$ for every $t \in\left[0, T_{0}\right]$. We claim that

$$
M(t) \geqslant w(t) \quad \forall t \in\left[0, T_{0}\right],
$$

from which 3.5 follows by letting $\varepsilon \rightarrow 0^{+}$. Let us assume for contradiction that $M(t)<w(t)$ for some $t \in\left[0, T_{0}\right]$, and set

$$
t_{0}:=\inf \left\{t \in\left[0, T_{0}\right]: v(r, t)<w(t) \forall r \in\left[r_{3}, r_{5}\right]\right\} .
$$

Since $M(0)>w(0)$, we have $t_{0}>0$. Moreover, due to the continuity of $v$ and $w$, there exists $r_{0} \in\left[r_{3}, r_{5}\right]$ such that $v\left(r_{0}, t_{0}\right)=w\left(t_{0}\right)$, and

$$
v\left(r, t_{0}\right)-w\left(t_{0}\right) \leqslant 0 \quad \forall r \in\left[r_{3}, r_{5}\right] .
$$

Since subcritical regions do not shrink, we have $v\left(r_{3}, t_{0}\right)<1$ and $v\left(r_{5}, t_{0}\right)<1$, while $w\left(t_{0}\right)>1$. This shows in particular that $r_{0} \neq r_{3}$ and $r_{0} \neq r_{5}$. Now we know that $r_{0}$ is a maximum point for the function $r \mapsto v\left(r, t_{0}\right)-w\left(t_{0}\right)$, and $r_{0}$ is contained in the open interval $\left(r_{3}, r_{5}\right)$, hence

$$
v_{r}\left(r_{0}, t_{0}\right)=0 \text { and } v_{r r}\left(r_{0}, t_{0}\right) \leqslant 0 .
$$

Let us now consider time derivatives. Since $v\left(r_{0}, t_{0}\right)=w\left(t_{0}\right)>1$, we can use (3.6). Exploiting also (3.7) and (3.9) we obtain

$$
v_{t}\left(r_{0}, t_{0}\right)-w_{t}\left(t_{0}\right)=\varphi^{\prime \prime}\left(v\left(r_{0}, t_{0}\right)\right) v_{r r}\left(r_{0}, t_{0}\right)-\frac{\varphi^{\prime}(v)}{r_{0}^{2}}+\frac{\varphi^{\prime}(1)}{r_{1}^{2}}>\varphi^{\prime \prime}\left(v\left(r_{0}, t_{0}\right)\right) v_{r r}\left(r_{0}, t_{0}\right) .
$$

Since $\varphi^{\prime \prime}\left(v\left(r_{0}, t_{0}\right)\right) \leqslant 0$, we conclude that

$$
v_{t}\left(r_{0}, t_{0}\right)-w_{t}\left(t_{0}\right)>\varphi^{\prime \prime}\left(v\left(r_{0}, t_{0}\right)\right) v_{r r}\left(r_{0}, t_{0}\right) \geqslant 0 .
$$

This implies that $v\left(r_{0}, t\right)-w(t)>0$ for every $t$ in a suitable right-hand neighborhood of $t_{0}$, which contradicts the definition of $t_{0}$.

\subsection{Proof of Theorem 2.4}

Let us set

$$
k_{1}:=n, \quad k_{2}:=1, \quad h_{1}:=n^{3}, \quad h_{2}:=-n^{2} .
$$

We claim that statements (1) and (2) of Theorem 2.4 hold true provided that $n$ is large enough. 
Statement (1). Let us set for simplicity $v_{0}(x, y):=\left|\nabla u_{0}(x, y)\right|^{2}$. Let us assume that

$$
v_{0 y}(0,0)>0 \text {. }
$$

Then the implicit function theorem implies that the set $v_{0}(x, y)>1$ can be represented, in a neighborhood of $(0,0)$, as $y>g(x)$, where $g$ is a suitable function defined in a neighborhood of $x=0$. Such a function satisfies

$$
\begin{gathered}
g(0)=0, \quad g^{\prime}(0)=-\frac{v_{0 x}(0,0)}{v_{0 y}(0,0)}, \\
g^{\prime \prime}(0)=-\frac{1}{v_{0 y}^{3}}\left\{v_{0 x}^{2} v_{0 y y}+v_{0 y}^{2} v_{0 x x}-2 v_{0 x} v_{0 y} v_{0 x y}\right\},
\end{gathered}
$$

where in 3.12 all partial derivatives of $v_{0}$ are computed at $(0,0)$. In particular $g$ is convex in a neighborhood of 0 if the right-hand side of 3.12 is positive.

From 2.12 we see that, up to higher order terms,

$$
u_{0 x}(x, y)=\frac{\sqrt{2}}{2}+2 k_{1} x+3 h_{1} x^{2}, \quad u_{0 y}(x, y)=\frac{\sqrt{2}}{2}+2 k_{2} y+3 h_{2} y^{2},
$$

hence

$$
v_{0}(x, y)=1+2 \sqrt{2}\left(k_{1} x+k_{2} y\right)+\left(4 k_{1}^{2}+3 \sqrt{2} h_{1}\right) x^{2}+\left(4 k_{2}^{2}+3 \sqrt{2} h_{2}\right) y^{2} .
$$

All the derivatives appearing in 3.11 and 3.12 can be easily computed. It follows that condition 3.11 is equivalent to $k_{2}>0$, while $g^{\prime \prime}(0)>0$ if and only if

$$
8 k_{1}^{2} k_{2}^{2}+3 \sqrt{2}\left(k_{1}^{2} h_{2}+k_{2}^{2} h_{1}\right)<0 .
$$

Both conditions are satisfied if the values of the parameters are given by 3.10 and $n$ is large enough.

Statement (2). Let us set for simplicity $v(x, y, t):=|\nabla u(x, y, t)|^{2}$. The statement will be proved if we show that

$$
v_{t}=2 u_{x} u_{t x}+2 u_{y} u_{t y}>0
$$

at $(x, y, t)=(0,0,0)$. We can then deduce the value of $v_{t}(0,0,0)$ from the Taylor expansion of $u_{0}(x, y)$.

In order to compute $u_{t}$, we recall that $u_{t}=\Psi_{1 x}+\Psi_{2 y}$, where

$$
\Psi_{1}:=\varphi^{\prime}\left(\left(u_{x}^{2}+u_{y}^{2}\right)^{1 / 2}\right) \frac{u_{x}}{\left(u_{x}^{2}+u_{y}^{2}\right)^{1 / 2}}, \quad \Psi_{2}:=\varphi^{\prime}\left(\left(u_{x}^{2}+u_{y}^{2}\right)^{1 / 2}\right) \frac{u_{y}}{\left(u_{x}^{2}+u_{y}^{2}\right)^{1 / 2}} .
$$

With some computations we obtain, up to higher order terms,

$$
\left(u_{x}^{2}+u_{y}^{2}\right)^{1 / 2}=1+\sqrt{2}\left(k_{1} x+k_{2} y\right)+\left[k_{1}^{2}+\frac{3 \sqrt{2}}{2} h_{1}\right] x^{2}+\left[k_{2}^{2}+\frac{3 \sqrt{2}}{2} h_{2}\right] y^{2}-2 k_{1} k_{2} x y,
$$

hence

$$
\varphi^{\prime}\left(\left(u_{x}^{2}+u_{y}^{2}\right)^{1 / 2}\right)=\varphi^{\prime}(1)+\left(k_{1} x+k_{2} y\right)^{2} \varphi^{\prime \prime \prime}(1),
$$


and therefore

$$
\begin{aligned}
\Psi_{1}= & \frac{\sqrt{2}}{2} \varphi^{\prime}(1)+\varphi^{\prime}(1)\left(k_{1} x-k_{2} y\right)+\frac{1}{2}\left[3 \varphi^{\prime}(1)\left(h_{1}-\sqrt{2} k_{1}^{2}\right)+\sqrt{2} \varphi^{\prime \prime \prime}(1) k_{1}^{2}\right] x^{2} \\
& +\frac{1}{2}\left[\varphi^{\prime}(1)\left(\sqrt{2} k_{2}^{2}-3 h_{2}\right)+\sqrt{2} \varphi^{\prime \prime \prime}(1) k_{2}^{2}\right] y^{2}+\sqrt{2}\left(\varphi^{\prime}(1)+\varphi^{\prime \prime \prime}(1)\right) k_{1} k_{2} x y .
\end{aligned}
$$

The expression for $\Psi_{2}$ is symmetric. It follows that, up to higher order terms,

$$
\begin{aligned}
u_{t}(x, y, 0)= & \left(k_{1}+k_{2}\right) \varphi^{\prime}(1) \\
& +\left\{\left(3 h_{1}+\sqrt{2} k_{1}\left(k_{2}-3 k_{1}\right)\right) \varphi^{\prime}(1)+\sqrt{2} \varphi^{\prime \prime \prime}(1) k_{1}\left(k_{1}+k_{2}\right)\right\} x \\
& +\left\{\left(3 h_{2}+\sqrt{2} k_{2}\left(k_{1}-3 k_{2}\right)\right) \varphi^{\prime}(1)+\sqrt{2} \varphi^{\prime \prime \prime}(1) k_{2}\left(k_{1}+k_{2}\right)\right\} y,
\end{aligned}
$$

hence

$$
v_{t}(0,0,0)=\varphi^{\prime}(1)\left\{3 \sqrt{2}\left(h_{1}+h_{2}\right)+4 k_{1} k_{2}-6 k_{1}^{2}-6 k_{2}^{2}\right\}+2 \varphi^{\prime \prime \prime}(1)\left(k_{1}+k_{2}\right)^{2} .
$$

From this expression it is easy to see that $v_{t}(0,0,0)>0$ if the values of the parameters are given by 3.10 and $n$ is large enough.

\subsection{Proof of Theorem 2.5}

Let $x_{\star} \in\left(x_{3}, x_{4}\right)$ be any point. We have to prove that

$$
v\left(x_{\star}, t\right)>0 \quad \forall t \in[0, T) .
$$

To this end we fix some notation. First of all we choose real numbers $x_{5}$ and $x_{6}$ such that $x_{3}<x_{5}<x_{\star}<x_{6}<x_{4}$. Then we consider the functions

$$
\psi(x):=\left(x-x_{5}\right)\left(x_{6}-x\right), \quad w(x, t):=e^{-\lambda t}\left(\delta^{2} \psi(x)+\delta \psi^{2}(x)\right),
$$

where $\lambda$ and $\delta$ are positive parameters. We claim that when $\lambda$ is large enough and $\delta$ is small enough we have

$$
v(x, t) \geqslant w(x, t) \quad \forall(x, t) \in\left[x_{5}, x_{6}\right] \times[0, T) .
$$

Since $w$ is positive in $\left(x_{5}, x_{6}\right) \times[0, T)$, and $x_{\star} \in\left(x_{5}, x_{6}\right)$, this is enough to prove (3.13). In order to prove (3.14 we first establish some properties of $w$.

Properties of $w$. Let us show that $w$ has the following properties:

(w1) $w \in C^{\infty}\left(\left[x_{5}, x_{6}\right] \times[0,+\infty)\right)$;

(w2) $w(x, t)>0$ for every $(x, t) \in\left(x_{5}, x_{6}\right) \times[0,+\infty)$;

(w3) $w_{x}\left(x_{5}, t\right)=\delta^{2} e^{-\lambda t} \psi^{\prime}\left(x_{5}\right)>0$ for every $t \geqslant 0$;

(w4) $w_{x}\left(x_{6}, t\right)=\delta^{2} e^{-\lambda t} \psi^{\prime}\left(x_{6}\right)<0$ for every $t \geqslant 0$;

(w5) if $\delta$ is small enough, then $w(x, 0)<v(x, 0)$ for every $x \in\left[x_{5}, x_{6}\right]$;

(w6) if $\delta$ is small enough, then $w(x, t)<c_{0}$ for every $(x, t) \in\left(x_{5}, x_{6}\right) \times[0,+\infty)$;

(w7) if $\delta$ is small enough and $\lambda$ is large enough, then

$$
w_{t}(x, t)<g(w(x, t)) w_{x x}(x, t) \quad \forall(x, t) \in\left(x_{5}, x_{6}\right) \times[0,+\infty) .
$$


Properties (w1) through (w4) easily follow from the definition of $w$ and $\psi$. Property (w5) follows from the fact that the infimum of $v(x, 0)$ over $x \in\left[x_{5}, x_{6}\right]$ is strictly positive due to (v3). Property (w6) is almost trivial. To prove (w7) we recall that $\psi^{\prime \prime}(x)=-2$, hence

$$
w_{t}(x, t)=-\lambda e^{-\lambda t}\left(\delta^{2} \psi(x)+\delta \psi^{2}(x)\right), \quad w_{x x}(x, t)=e^{-\lambda t}\left(2 \delta\left[\psi^{\prime}(x)\right]^{2}-4 \delta \psi(x)-2 \delta^{2}\right) .
$$

After plugging these identities in 3.15, it remains to prove that

$$
-\lambda\left(\delta \psi+\psi^{2}\right)<g(w)\left(2\left[\psi^{\prime}\right]^{2}-4 \psi-2 \delta\right) \quad \forall(x, t) \in\left(x_{5}, x_{6}\right) \times[0,+\infty) .
$$

To this end we fix once for all two real numbers $x_{7}$ and $x_{8}$ such that $x_{5}<x_{7}<x_{8}<x_{6}$, and

$$
\inf \left\{2\left[\psi^{\prime}(x)\right]^{2}-4 \psi(x): x \in\left[x_{5}, x_{7}\right] \cup\left[x_{8}, x_{6}\right]\right\}>0 .
$$

This is possible because at the endpoints of the interval $\left[x_{5}, x_{6}\right]$ one has $\psi=0$ and $\psi^{\prime} \neq 0$. Now we distinguish two cases. When $x \in\left[x_{5}, x_{7}\right] \cup\left[x_{8}, x_{6}\right]$ the left-hand side of 3.16 is negative, while the right-hand side is positive provided that $\delta$ is small enough, independently of $x$. When $x \in\left[x_{7}, x_{8}\right]$ the right-hand side may be negative, but also the left-hand side is strictly negative because in this interval $\psi$ is bounded from below by a positive constant. In other words, in $\left[x_{7}, x_{8}\right] \times[0,+\infty)$ inequality 3.16 holds true if we choose

$$
\lambda>\sup \left\{\frac{g(w(x, t))(4 \psi(x)+2 \delta)}{\delta \psi(x)+\psi^{2}(x)}:(x, t) \in\left[x_{7}, x_{8}\right] \times[0,+\infty)\right\} .
$$

We point out that the supremum is finite. This completes the proof of 3.16).

Proof of (3.14). Let us choose $\delta, \lambda>0$ such that $w$ satisfies (w1) through (w7). Now we argue more or less as in the proof of the classical comparison results. Let us assume that (3.14) is not true, and let us set

$$
t_{0}:=\inf \left\{t \in[0, T): \exists x \in\left[x_{5}, x_{6}\right] \text { such that } v(x, t)-w(x, t)<0\right\} .
$$

From (w5) we have $t_{0}>0$. Moreover, from the definition of $t_{0}$ it follows that

$$
v(x, t)-w(x, t) \geqslant 0 \quad \forall(x, t) \in\left[x_{5}, x_{6}\right] \times\left[0, t_{0}\right] .
$$

Finally, from the continuity of $v$ and $w$ we deduce that there exists $x_{0} \in\left[x_{5}, x_{6}\right]$ such that $v\left(x_{0}, t_{0}\right)-$ $w\left(x_{0}, t_{0}\right)=0$.

We claim that $x_{0} \neq x_{5}$. Indeed let us assume for contradiction that $x_{0}=x_{5}$. Then $w\left(x_{0}, t_{0}\right)=0$, hence also $v\left(x_{0}, t_{0}\right)=0$. By (v2) it follows that $x_{0}$ is a minimum point for the function $x \mapsto v\left(x, t_{0}\right)$. By (v4) we therefore have $v_{x}\left(x_{0}, t_{0}\right)=0$. Keeping (w3) in mind, we deduce that

$$
(v-w)_{x}\left(x_{0}, t_{0}\right)=v_{x}\left(x_{0}, t_{0}\right)-w_{x}\left(x_{0}, t_{0}\right)<0 .
$$

On the other hand, from 3.17 we know that also $x_{0}=x_{5}$ is a minimum point for the function $x \mapsto v\left(x, t_{0}\right)-w\left(x, t_{0}\right)$ restricted to the interval $\left[x_{5}, x_{6}\right]$. Since the minimum point is the left endpoint of the interval, we deduce that $(v-w)_{x}\left(x_{0}, t_{0}\right) \geqslant 0$, which contradicts 3.18 .

In a symmetric way we prove that $x_{0} \neq x_{6}$. So we are left with the case $t_{0}>0$ and $x_{0} \in\left(x_{5}, x_{6}\right)$. In this case by (w2) and (w6) we have $0<v\left(x_{0}, t_{0}\right)=w\left(x_{0}, t_{0}\right)<c_{0}$, hence both $v$ and $w$ are 
smooth in a neighborhood of this point and satisfy (v5) and (w7), respectively. In particular, since $x_{0}$ is always a minimum point of the function $x \mapsto v\left(x, t_{0}\right)-w\left(x, t_{0}\right)$, and now $x_{0} \in\left(x_{5}, x_{6}\right)$, we have

$$
v_{x}\left(x_{0}, t_{0}\right)=w_{x}\left(x_{0}, t_{0}\right) \text { and } v_{x x}\left(x_{0}, t_{0}\right) \geqslant w_{x x}\left(x_{0}, t_{0}\right) .
$$

Let us now consider the time derivatives. On the one hand, $(v-w)_{t}\left(x_{0}, t_{0}\right) \leqslant 0$ from (3.17). On the other hand, from (v5), (w7), and (3.19) we have

$$
\begin{aligned}
(v-w)_{t}\left(x_{0}, t_{0}\right)> & g\left(v\left(x_{0}, t_{0}\right)\right) v_{x x}\left(x_{0}, t_{0}\right)-g\left(w\left(x_{0}, t_{0}\right)\right) w_{x x}\left(x_{0}, t_{0}\right) \\
& =g\left(w\left(x_{0}, t_{0}\right)\right)\left(v_{x x}\left(x_{0}, t_{0}\right)-w_{x x}\left(x_{0}, t_{0}\right)\right) \geqslant 0 .
\end{aligned}
$$

This rules out the last case and completes the proof of (3.14).

\subsection{Proof of Theorem 2.6}

The strategy is similar to the proof of Theorem 2.5. The main difference is that in this case we have to cope with moving domains.

Let $\left(r_{\star}, t_{\star}\right)$ be any point of $\mathcal{D}$. We have to prove that

$$
v\left(r_{\star}, t_{\star}\right)>0 .
$$

To this end we fix some notation. First of all it is not difficult to see that there exist real numbers $r_{5}, r_{6}, k$ such that

$$
|k|<G \sqrt{A}, \quad r_{1}<r_{5}+k t_{\star}<r_{\star}<r_{6}+k t_{\star}<r_{2} .
$$

Then we consider the set

$$
\mathcal{D}_{\star}:=\left\{(r, t) \in\left(r_{1}, r_{2}\right) \times[0, T): t \leqslant t_{\star}, r_{5}+k t \leqslant r \leqslant r_{6}+k t\right\} \subseteq \mathcal{D} .
$$

We refer to Figure 3 for a representation of $\mathcal{D}_{\star}$ (corresponding in that case to some $k<0$ ) and its relation to $\left(r_{\star}, t_{\star}\right)$ and $\mathcal{D}$. Note that the slope of the slanted lines bounding $\mathcal{D}_{\star}$ is larger than the slope of the slanted lines limiting $\mathcal{D}$. This is just because $|k|<G \sqrt{A}$.
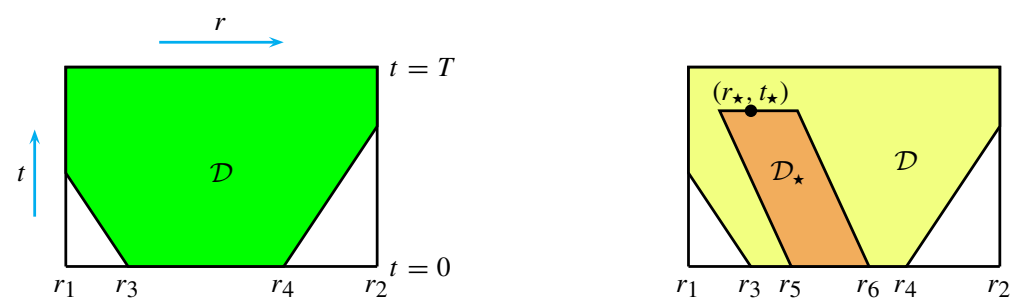

FIG. 3. The sets $\mathcal{D}$ and $\mathcal{D} \star$

Due to this inequality, there exists $\varepsilon_{0} \in(0, \min \{1, G, A / 2\})$ such that

$$
|k|<\left(1-\varepsilon_{0}\right)\left(G-\varepsilon_{0}\right) \sqrt{A-2 \varepsilon_{0}} .
$$

From now on, $\varepsilon_{0}$ is a fixed positive constant. Due to 2.13 there also exists $c_{2} \in\left(0, c_{0}\right)$ such that

$$
g(\sigma) \geqslant\left(G-\varepsilon_{0}\right) \sqrt{\sigma} \quad \forall \sigma \in\left(0, c_{2}\right) .
$$


Finally we consider the functions

$$
\psi(r):=\left(r-r_{5}\right)\left(r_{6}-r\right), \quad w(r, t):=\delta^{3} \psi(r-k t)+\delta[\psi(r-k t)]^{3 / 2},
$$

where $\delta$ is a positive parameter. We claim that when $\delta$ is small enough we have

$$
v(r, t) \geqslant w(r, t) \quad \forall(r, t) \in \mathcal{D}_{\star} .
$$

This inequality, applied with $(r, t)=\left(r_{\star}, t_{\star}\right)$, implies 3.20 . In order to prove 3.23$)$ we first establish some properties of $w$.

Properties of $w$. Let $\operatorname{Int}\left(\mathcal{D}_{\star}\right)$ denote the set of points $(r, t) \in \mathcal{D}_{\star}$ with $r_{5}+k t<r<r_{6}+k t$. Let us show that $w$ has the following properties:

(w1) $w \in C^{1}\left(\mathcal{D}_{\star}\right) \cap C^{\infty}\left(\operatorname{Int}\left(\mathcal{D}_{\star}\right)\right)$;

(w2) $w(r, t)>0$ for every $(r, t) \in \operatorname{Int}\left(\mathcal{D}_{\star}\right)$;

(w3) $w_{r}\left(r_{5}+k t, t\right)=\delta^{3} \psi^{\prime}\left(r_{5}\right)>0$ for every $t \in\left[0, t_{\star}\right]$;

(w4) $w_{r}\left(r_{6}+k t, t\right)=\delta^{3} \psi^{\prime}\left(r_{6}\right)<0$ for every $t \in\left[0, t_{\star}\right]$;

(w5) if $\delta$ is small enough, then $w(r, 0)<v(r, 0)$ for every $r \in\left[r_{5}, r_{6}\right]$;

(w6) if $\delta$ is small enough, then $w(r, t)<c_{2}$ for every $(r, t) \in \mathcal{D}_{\star}$;

(w7) if $\delta$ is small enough, then

$$
w_{t}<g(w)\left\{w_{r r}+f\left(r, t, w, w_{r}\right)+A\right\} \quad \forall(r, t) \in \operatorname{Int}\left(\mathcal{D}_{\star}\right) .
$$

Properties (w1) through (w4) easily follow from the definition of $w$ and $\psi$. Property (w5) follows from the fact that the infimum of $v(r, 0)$ over $r \in\left[r_{5}, r_{6}\right]$ is strictly positive due to (v3). Property (w6) is almost trivial. To prove (w7) we recall that $\psi^{\prime \prime}(r)=-2$, hence (for simplicity we set $y:=r-k t$, and we observe that $\left.y \in\left[r_{5}, r_{6}\right]\right)$

$$
\begin{aligned}
w_{t}(r, t) & =-k \delta^{3} \psi^{\prime}(y)-\frac{3}{2} k \delta[\psi(y)]^{1 / 2} \psi^{\prime}(y), \\
w_{r}(r, t) & =\delta^{3} \psi^{\prime}(y)+\frac{3}{2} \delta[\psi(y)]^{1 / 2} \psi^{\prime}(y), \\
w_{r r}(r, t) & =-2 \delta^{3}-3 \delta[\psi(y)]^{1 / 2}+\frac{3}{4} \delta[\psi(y)]^{-1 / 2}\left[\psi^{\prime}(y)\right]^{2} .
\end{aligned}
$$

When $\delta \rightarrow 0^{+}$we see that $w$ and $w_{r}$ tend to zero uniformly in $\mathcal{D}_{\star}$. Thanks to 2.14 we therefore have

$$
\left|f\left(r, t, w(r, t), w_{r}(r, t)\right)\right| \leqslant \varepsilon_{0} \quad \forall(r, t) \in \mathcal{D}_{\star},
$$

provided that $\delta$ is small enough. In an analogous way we also have

$$
w_{r r}(r, t)>\frac{3}{4} \delta[\psi(y)]^{-1 / 2}\left[\psi^{\prime}(y)\right]^{2}-\varepsilon_{0}
$$

provided that $\delta$ is small enough. It follows that

$$
w_{r r}+f\left(r, t, w, w_{r}\right)+A>A-2 \varepsilon_{0}+\frac{3}{4} \delta[\psi(y)]^{-1 / 2}\left[\psi^{\prime}(y)\right]^{2}>0
$$


in $\operatorname{Int}\left(\mathcal{D}_{\star}\right)$. Moreover, from 3.22), (w2) and (w6) we have

$$
g(w(r, t)) \geqslant\left(G-\varepsilon_{0}\right) \sqrt{w(r, t)} \quad \forall(r, t) \in \operatorname{Int}\left(\mathcal{D}_{\star}\right),
$$

and in conclusion

$$
g(w)\left\{w_{r r}+f\left(r, t, w, w_{r}\right)+A\right\}>\left(G-\varepsilon_{0}\right) \sqrt{w}\left\{A-2 \varepsilon_{0}+\frac{3}{4} \delta \frac{\left[\psi^{\prime}(y)\right]^{2}}{[\psi(y)]^{1 / 2}}\right\} .
$$

Therefore inequality (3.24) is proved if we show that

$$
\delta^{3}\left|k \psi^{\prime}\right|+\frac{3}{2} \delta\left|k \psi^{\prime}\right| \sqrt{\psi} \leqslant\left(G-\varepsilon_{0}\right) \sqrt{\delta^{3} \psi+\delta[\psi]^{3 / 2}}\left\{A-2 \varepsilon_{0}+\frac{3}{4} \delta \frac{\left[\psi^{\prime}\right]^{2}}{\sqrt{\psi}}\right\},
$$

where the argument of $\psi$ and $\psi^{\prime}$ is any $y \in\left(r_{5}, r_{6}\right)$. Let us consider the right-hand side of 3.25 multiplied by $1-\varepsilon_{0}$. Applying the inequality between the arithmetic and geometric means, and recalling 3.21, we obtain

$$
\begin{aligned}
\left(1-\varepsilon_{0}\right) \cdot(\text { right-hand side }) & \geqslant\left(1-\varepsilon_{0}\right)\left(G-\varepsilon_{0}\right) \sqrt{\delta[\psi]^{3 / 2}} \cdot\left\{A-2 \varepsilon_{0}+\frac{3}{4} \delta \frac{\left[\psi^{\prime}\right]^{2}}{\sqrt{\psi}}\right\} \\
& \geqslant\left(1-\varepsilon_{0}\right)\left(G-\varepsilon_{0}\right) \sqrt{\delta[\psi]^{3 / 2}} \cdot 2\left[\left(A-2 \varepsilon_{0}\right) \cdot \frac{3}{4} \delta \frac{\left[\psi^{\prime}\right]^{2}}{\sqrt{\psi}}\right]^{1 / 2} \\
& =\left(1-\varepsilon_{0}\right)\left(G-\varepsilon_{0}\right) \sqrt{A-2 \varepsilon_{0}} \cdot \delta\left|\psi^{\prime}\right| \sqrt{\psi} \cdot \sqrt{3} \geqslant \frac{3}{2} \delta\left|k \psi^{\prime}\right| \sqrt{\psi} .
\end{aligned}
$$

In order to prove 3.25 it is therefore enough to show that

$$
\delta^{3}\left|k \psi^{\prime}\right| \leqslant \varepsilon_{0}\left(G-\varepsilon_{0}\right) \sqrt{\delta^{3} \psi+\delta[\psi]^{3 / 2}}\left\{A-2 \varepsilon_{0}+\frac{3}{4} \delta \frac{\left[\psi^{\prime}\right]^{2}}{\sqrt{\psi}}\right\},
$$

which in turn is true if we show that

$$
\delta^{3 / 2}\left|k \psi^{\prime}\right| \leqslant \varepsilon_{0}\left(G-\varepsilon_{0}\right) \sqrt{\psi}\left\{A-2 \varepsilon_{0}+\frac{3}{4} \delta \frac{\left[\psi^{\prime}\right]^{2}}{\sqrt{\psi}}\right\} .
$$

To this end, we fix once for all two real numbers $r_{7}$ and $r_{8}$ such that

$$
r_{5}<r_{7}<\frac{r_{5}+r_{6}}{2}<r_{8}<r_{6}
$$

When $y \in\left(r_{5}, r_{7}\right] \cup\left[r_{8}, r_{6}\right)$ we see that $\left|\psi^{\prime}(y)\right|$ is bounded from below by a positive constant. Therefore

$$
\delta^{3 / 2}\left|k \psi^{\prime}(y)\right| \leqslant \varepsilon_{0}\left(G-\varepsilon_{0}\right) \frac{3}{4} \delta\left[\psi^{\prime}(y)\right]^{2}
$$

provided that $\delta$ is small enough. When $y \in\left[r_{7}, r_{8}\right]$ we see that $\psi(y)$ is bounded from below by a positive constant, hence in this case

$$
\delta^{3 / 2}\left|k \psi^{\prime}(y)\right| \leqslant \varepsilon_{0}\left(G-\varepsilon_{0}\right)\left(A-2 \varepsilon_{0}\right) \sqrt{\psi(y)}
$$


provided that $\delta$ is small enough. This completes the proof of 3.25 and shows that $w$ satisfies (w7) whenever $\delta$ is small enough.

Proof of (3.23). The argument is analogous to the proof of the corresponding inequality in Theorem 2.5 Let us choose $\delta>0$ such that $w$ satisfies (w1) through (w7). Let us assume that (3.23) is not true, and let us set

$$
t_{0}:=\inf \left\{t \in\left[0, t_{\star}\right]: \exists r \in\left[r_{5}+k t, r_{6}+k t\right] \text { such that } v(r, t)-w(r, t)<0\right\} .
$$

From (w5) we have $t_{0}>0$. Moreover, from the definition of $t_{0}$ we have

$$
v(r, t)-w(r, t) \geqslant 0 \quad \forall(r, t) \in \mathcal{D}_{\star} \text { with } t \leqslant t_{0} .
$$

Finally, due to the continuity of $v$ and $w$, we also deduce that there exists $r_{0} \in\left[r_{5}+k t_{0}, r_{6}+k t_{0}\right]$ such that $v\left(r_{0}, t_{0}\right)-w\left(r_{0}, t_{0}\right)=0$.

We claim that $r_{0} \neq r_{5}+k t_{0}$. Indeed let us assume for contradiction that $r_{0}=r_{5}+k t_{0}$. Then $w\left(r_{0}, t_{0}\right)=0$, hence also $v\left(r_{0}, t_{0}\right)=0$. By (v2) it follows that $r_{0}$ is a minimum point for the function $r \mapsto v\left(r, t_{0}\right)$. By (v4) we therefore have $v_{r}\left(r_{0}, t_{0}\right)=0$. Taking (w3) into account, we deduce that

$$
(v-w)_{r}\left(r_{0}, t_{0}\right)=v_{r}\left(r_{0}, t_{0}\right)-w_{r}\left(r_{0}, t_{0}\right)<0 .
$$

On the other hand, from (3.26) we also know that $r_{0}=r_{5}+k t_{0}$ is a minimum point for the function $r \mapsto v\left(r, t_{0}\right)-w\left(r, t_{0}\right)$ restricted to the interval $\left[r_{5}+k t_{0}, r_{6}+k t_{0}\right]$. Since the minimum point is the left endpoint of the interval, we deduce that $(v-w)_{r}\left(r_{0}, t_{0}\right) \geqslant 0$, which contradicts (3.27).

In a symmetric way we prove that $r_{0} \neq r_{6}+k t_{0}$. So we are left with the case in which $t_{0}>0$ and $r_{0} \in\left(r_{5}+k t_{0}, r_{6}+k t_{0}\right)$. In this case $0<v\left(r_{0}, t_{0}\right)=w\left(r_{0}, t_{0}\right)<c_{2}$, hence both $v$ and $w$ are smooth in a neighborhood of this point and satisfy (v5) and (w7), respectively. In particular, since $r_{0}$ is always a minimum point of the function $r \mapsto v\left(r, t_{0}\right)-w\left(r, t_{0}\right)$, and now $r_{0}$ is in the interior of the interval $\left(r_{5}+k t_{0}, r_{6}+k t_{0}\right)$, we have

$$
v_{r}\left(r_{0}, t_{0}\right)=w_{r}\left(r_{0}, t_{0}\right) \quad \text { and } \quad v_{r r}\left(r_{0}, t_{0}\right) \geqslant w_{r r}\left(r_{0}, t_{0}\right) .
$$

Let us now consider the time derivatives. On the one hand, $(v-w)_{t}\left(r_{0}, t_{0}\right) \leqslant 0$ from 3.26 . On the other hand, from (v5), (w7), and (3.28), at the point $\left(r_{0}, t_{0}\right)$ we have

$$
\begin{aligned}
(v-w)_{t} & >g(v)\left\{v_{r r}+f\left(r, t, v, v_{r}\right)+A\right\}-g(w)\left\{w_{r r}+f\left(r, t, w, w_{r}\right)+A\right\} \\
& =g(w)\left\{v_{r r}+f\left(r, t, w, w_{r}\right)+A\right\}-g(w)\left\{w_{r r}+f\left(r, t, w, w_{r}\right)+A\right\} \\
& =g(w)\left(v_{r r}-w_{r r}\right) \geqslant 0 .
\end{aligned}
$$

This rules out the last case and completes the proof of 3.23 .

\section{REFERENCES}

1. Amann, H. Time-delayed Perona-Malik type problems. Acta Math. Univ. Comenian. (N.S.) 76 (2007), 15-38. Zbl 1132.68067 MR 2331050

2. Bellettini, G., \& Fusco, G. The $\Gamma$-limit and the related gradient flow for singular perturbation functionals of Perona-Malik type. Trans. Amer. Math. Soc. 360 (2008), 4929-4987. Zbl 1191.49010 MR 2403710 
3. Bellettini, G., Novaga, M., \& Paolini, E. Global solutions to the gradient flow equation of a nonconvex functional. SIAM J. Math. Anal. 37 (2006), 1657-1687. Zbl 1109.35050 MR 2215602

4. Bellettini, G., Novaga, M., Paolini, M., \& Tornese, C. Convergence of discrete schemes for the Perona-Malik equation. J. Differential Equations 245 (2008), 892-924. Zbl 1155.35002 MR 2427400

5. CAtté, F., Lions, P.-L., Morel, J.-M., \& Coll, T. Image selective smoothing and edge detection by nonlinear diffusion. SIAM J. Numer. Anal. 29 (1992), 182-193. Zbl 0746.65091 MR 1149092

6. Chen, Y., \& ZhAng, K. Young measure solutions of the two-dimensional Perona-Malik equation in image processing. Comm. Pure Appl. Anal. 5 (2006), 615-635. Zbl 1141.35012 MR 2217594

7. EsedoḠLU, S. Stability properties of the Perona-Malik scheme. SIAM J. Numer. Anal. 44 (2006), 12971313. Zbl 1127.94002 MR 2231865

8. Esedō̄LU, S., \& GREer, J. B. Upper bounds on the coarsening rate of discrete, ill-posed nonlinear diffusion equations. Comm. Pure Appl. Math. 62 (2009), 57-81. Zbl 1161.35057 MR 2460266

9. Ghisi, M., \& Gobbino, M. Gradient estimates for the Perona-Malik equation. Math. Ann. 337 (2007), 557-590. Zbl 1130.35081 MR 2274543

10. Ghisi, M., \& Gobbino, M. A class of local classical solutions for the one-dimensional Perona-Malik equation. Trans. Amer. Math. Soc. 361 (2009), 6429-6446. Zbl pre05638196 MR 2538599

11. Ghisi, M., \& GobBino, M. An example of global classical solution for the Perona-Malik equation. Comm. Partial Differential Equations, to appear.

12. Gobbino, M. Entire solutions of the one-dimensional Perona-Malik equation. Comm. Partial Differential Equations 32 (2007), 719-743. Zbl 1134.35062 MR 2334830

13. Guidotti, P. A new nonlocal nonlinear diffusion of image processing, J. Differential Equations 246 (2009), 4731-4742. Zbl 1170.35450 MR 2523301

14. Guidotti, P., \& Lambers, J. V. Two new nonlinear nonlocal diffusions for noise reduction. J. Math. Imaging Vision 33 (2009), 25-37. MR 2471042

15. KAWOHL, B., \& KUTEV, N. Maximum and comparison principle for one-dimensional anisotropic diffusion. Math. Ann. 311 (1998), 107-123. Zbl 0909.35025 MR 1624275

16. Kichenassamy, S. The Perona-Malik paradox. SIAM J. Appl. Math. 57 (1997), 1328-1342. Zbl 0887.35071 MR 1470926

17. Perona, P., \& Malik, J. Scale space and edge detection using anisotropic diffusion, IEEE Trans. Pattern Anal. Mach. Intell. 12 (1990), 629-639.

18. TAHERI, S., TANG, Q., \& ZHANG, K. Young measure solutions and instability of the one-dimensional Perona-Malik equation. J. Math. Anal. Appl. 308 (2005), 467-490. Zbl 1081.35056 MR 2150102

19. Weickert, J. Anisotropic Diffusion in Image Processing. European Consortium for Mathematics in Industry, Teubner, Stuttgart (1998). Zbl 0886.68131 MR 1666943

20. ZhANG, K. Existence of infinitely many solutions for the one-dimensional Perona-Malik model. Calc. Var. Partial Differential Equations 26 (2006), 171-199. Z Zbl 1096.35067| MR 2222243 